Research Article

\title{
Current Driver Design for Electromagnetic Coil Using Adaptive Active Disturbance Rejection Control
}

\author{
Hongtao Zhu (D), Xiaoting Rui $(\mathbb{D}$, Fufeng Yang, and Wei Zhu \\ Institute of Launch Dynamics, Nanjing University of Science and Technology, Nanjing 210094, China \\ Correspondence should be addressed to Xiaoting Rui; ruixt@163.net
}

Received 12 July 2020; Revised 11 December 2020; Accepted 24 December 2020; Published 5 January 2021

Academic Editor: Stefano Manzoni

Copyright (c) 2021 Hongtao Zhu et al. This is an open access article distributed under the Creative Commons Attribution License, which permits unrestricted use, distribution, and reproduction in any medium, provided the original work is properly cited.

\begin{abstract}
An adaptive active disturbance rejection controller is used in the current driver design of the electromagnetic coil. Extended state observer of the $1^{\text {st }}$-order system is adopted for disturbance observation of ADRC. The supervised recursive least squares method is proposed for real-time parameters estimation, in which the excitation signal variance is used to trigger the parameter estimator. The experimental results demonstrate that ADRC combined with real-time parameter estimation simplifies the parameter tuning and improves the parameter adaptive ability.
\end{abstract}

\section{Introduction}

Electromagnetic coil, which is usually used to generate a magnetic field, is an important part of many devices, such as electrovalves and motor. The accuracy and speed of the current response have a great influence on the function of those devices. Also, the electromagnetic coil with iron core is an important component of magnetorheological (MR) damper Figure 1, which has been applied in many fields, such as structural vibration reduction [1-3] and vehicle suspension $[4,5]$. The main motivation of this paper is to design a new digital current driver for the MR damper.

The parameters of the coil of the MR damper will be affected by temperature and other factors, which will affect the control effect of the coil current. In this paper, an adaptive active disturbance rejection control (AADRC) is used to design the current driver of MR damper, and a supervised recursive least square method is proposed for online parameters estimation, which makes the system more adaptable to parameters change.

In the past decades, online parameter identification has been widely used in fault detection [6] and adaptive control [7] and the recursive least square (RLS) method is one of the most widely used online parameter identification algorithms [8]. The main problem is that the estimator will wind-up and the estimation results are unsatisfactory when there is no or little excitation [9]. There are three main methods to solve this problem: (1) providing additional excitation; (2) double controllers [10, 11]; and (3) ensuring that the estimator works when the excitation is enough [12-14]. The first method is the simplest, but it cannot achieve satisfactory results. The reason is that, on the one hand, when the excitation is too small, the signal-to-noise ratio of the system output signal is low; on the other hand, too much additional excitation will affect the control performance of the closedloop system. Therefore, this method is seldom used in practical engineering. The second method makes the closedloop system identifiable by switching between two different controllers in the system control. Hjalmarsson discussed some problems brought about by this method in [15]. The third method does not need to provide additional incentives in the loop, nor does it need to change the structure of the controller. And it is a simple and effective method. However, this method needs to detect excitation. Transient detection was used to trigger the adaptive controller by Moraal [14]. The high-pass filter is utilized to detect excitation in [12]. These two methods are not suitable for the case where the excitation is a random signal. In this paper, a supervised recursive least squares (SRLS) method is proposed, in which the excitation is detected by estimating the variance of the excitation, and it can achieve good results. 

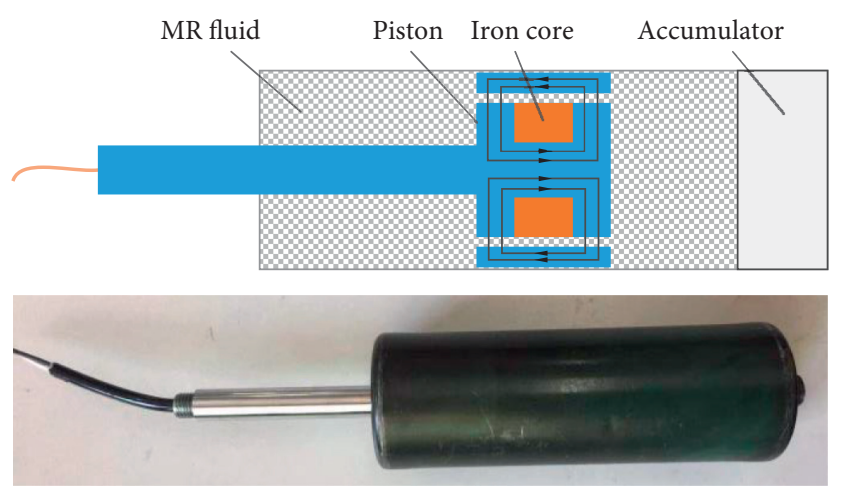

FIgURE 1: Structure of MR damper.

ADRC is widely taken into application in various fields. Sun and Gao [16] apply ADRC to the design of H-bridge DC-DC power converter. ADRC is applied to uncertain multivariable systems with time delay in [17]. Zheng et al. [18] adopt ADRC for MEMS gyroscope. Linear ObserverBased ADRC is adopted in the omnidirectional mobile robot by Sira-Ramírez et al. [19] and the results are achieved in the laboratory. It is also introduced in hydraulic servo systems by Yao and Deng [20] and the experimental results demonstrate the high tracking performance of this method. In this paper, ADRC is introduced to improve the efficiency of controller parameter tuning by separation of ADRC design process [21, 22].

This paper has the following contributions: (1) a supervised recursive least squares method is proposed, which makes the estimator enabled by excitation variance, and the process is simple and effective; (2) the ADRC is adopted to improve the efficiency of parameter tuning in the controller design process; and (3) an AADRC method is proposed to improve the process parameters adaptability, by combining the online parameter estimation with ADRC.

This paper is organized as follows. In Section 2, a brief description of system configuration and modeling is presented. The process is introduced in detail in Section 3. In Section 4, the experimental results and the comparisons with PI controller is given. Moreover, a conclusion will be drawn in Section 5.

\section{System Configuration and Modeling}

2.1. Modeling for System. The excitation coil is considered as an inductance-resistance model, which is expressed by the following differential equations:

$$
I_{o}+\frac{L}{R} \frac{\mathrm{d} I_{o}}{\mathrm{~d} t}=\frac{V_{s}}{R} u .
$$

In equation (1), $L$ denotes coil inductance and $R$ denotes coil resistance. $I_{o}$ is the current output; $V_{s}$ is the power supply voltage and $u$ is the control output. If $u$ is -1 , the PWM duty is $0 \%$; if $u$ is 0 , the output PWM duty is $50 \%$; and if $u$ is 1 , the PWM duty is $100 \%$. Since the bootstrap circuit cannot keep the duty cycle $100 \%$ for a long time, the output value is chosen in $[-0.95,0.95]$.
Figure 2 shows the step response of the uncontrolled system (supply voltage is $24 \mathrm{~V}$ ), and Figure 2(a) shows the system step input (PWM wave with a duty cycle of 0.25 ). Form Figure 2, the circuit parameters, shown in Table 1, can be obtained by first-order system approximation method [23].

The coil resistance generally varies linearly with temperature and the form is given by the following equation:

$$
R(T)=k_{R} T+R_{0}
$$

where $k_{R}$ is the coil resistance temperature coefficient; $T$ is the coil temperature $\left({ }^{\circ} \mathrm{C}\right)$; and $R_{0}$ is the coil resistance at $0^{\circ} \mathrm{C}$.

The coil resistance of the MR damper at different temperatures was measured by experiment as shown in Figure 3. The results show that the coil resistance is about $5.57 \Omega$ at room temperature $\left(25^{\circ} \mathrm{C}\right)$; when the temperature changes from $-40^{\circ} \mathrm{C}$ to $125^{\circ} \mathrm{C}\left(-40^{\circ} \mathrm{C} \sim 125^{\circ} \mathrm{C}\right.$ is the working temperature of magnetorheological fluid), the coil resistance is expected to change by $3.1 \Omega$. Compared with resistance at room temperature, the resistance decreases by $21.9 \%$ at $-40^{\circ} \mathrm{C}$ and increases by $33.8 \%$ at $125^{\circ} \mathrm{C}$.

\section{Controller Design}

The block diagram of the adaptive ADRC controller designed in this paper is shown in Figure 4.

It can be seen from the figure that the AADRC designed in this paper is mainly composed of tracking differentiator (TD), extended state observer (ESO), and online parameter estimator (online $\mathrm{PE}$ ). The role of $\mathrm{TD}$ is to provide the differential signal of the desired current $I_{e}$; ESO is used to observe the system disturbance; the online parameter estimator is used to estimate the system perimeters; and $\widehat{\theta}$ is the estimated parameters vector which is used to adjust the controller gain. The controller output $u$ is given as

$$
u=K_{f} \dot{I}_{e}+K_{p} e+u_{\mathrm{eso}}
$$

where $K_{p}$ is the feedback control proportional gain coefficient; $K_{f}$ is the feedforward control gain coefficient; $e=$ $I_{o}-I_{e}$ is defined as control error; $u_{\text {eso }}$ represents the compensate from ESO and it is defined as

$$
u_{\text {eso }}=-\frac{L}{V_{s}}\left(z_{2}-\frac{R}{L} I_{o}\right) \text {. }
$$

3.1. Extended State Observer. The ESO is the core of ADRC. The following equation gives the form of the linear ESO of the $1^{\text {st }}$-order system:

$$
\left\{\begin{array}{l}
\dot{z}_{1}=z_{2}+v+L_{1}\left(I_{o}-z_{1}\right), \\
\dot{z}_{2}=L_{2}\left(I_{o}-z_{1}\right) .
\end{array}\right.
$$

In equation (5), $z_{1}$ and $z_{2}$ are the state variables of the ESO and $L=\left[\begin{array}{ll}L_{1} & L_{2}\end{array}\right]^{T}$ is the ESO gain matrix. Reference [24] gives the pole configuration method to obtain the observer gain of ESO. For the above ESO in equation (5), the characteristic equation is given as follows: 


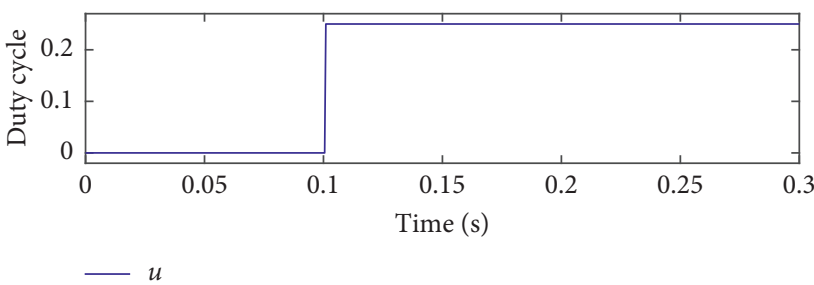

(a)

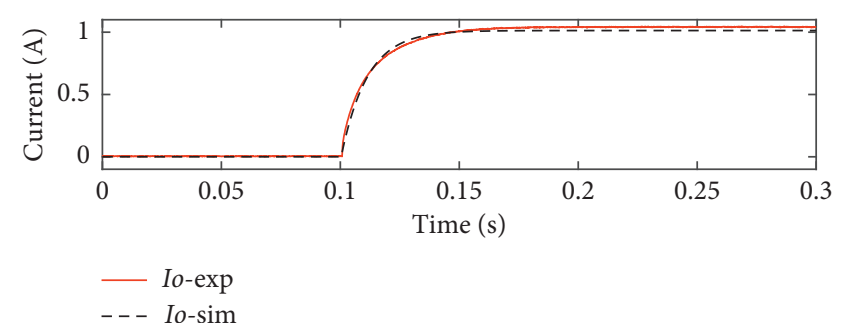

(b)

Figure 2: The step response of the uncontrolled system.

TABle 1: Parameters of MR damper coil $\left(25^{\circ} \mathrm{C}\right)$.

\begin{tabular}{lcc}
\hline Parameters & Value & Unit \\
\hline$R$ & 5.57 & $\Omega$ \\
$L$ & 0.0652 & $H$ \\
\hline
\end{tabular}

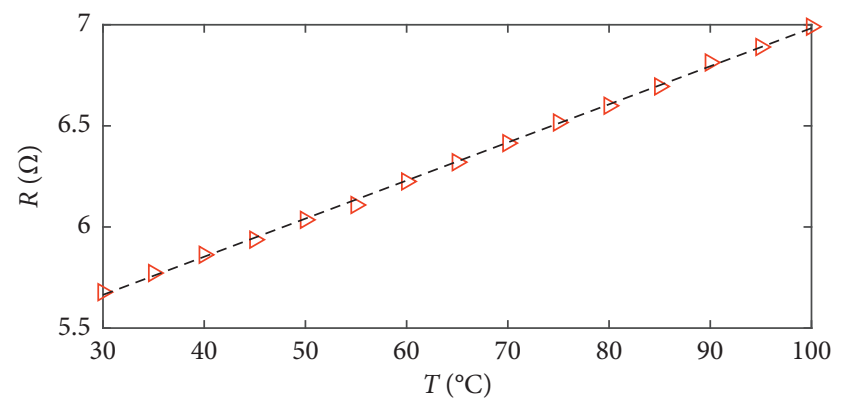

$\triangleright$ Experiment data

- - - Fitting data

FIgURE 3: Coil resistance varies with temperature.

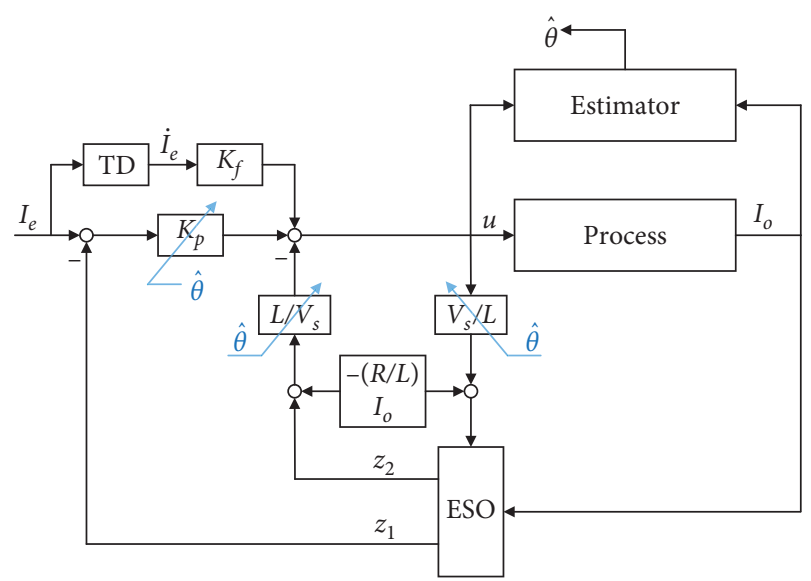

FIGURE 4: Block diagram of the controller.

$$
s^{2}+L_{1} s+L_{2}=\left(s-s_{1}\right)\left(s-s_{2}\right) .
$$

In equation (6), $s_{1}$ and $s_{2}$ are the poles of the characteristic equation. For the sake of simplicity, $s_{1}=s_{2}=-\sigma$ is taken in this paper and $\sigma$ is a positive real number. The gain matrix $L$ is given in the following equation:

$$
L=\left[\begin{array}{cc}
2 \sigma & \sigma^{2}
\end{array}\right]^{T}
$$

The discrete form of equation (5) is

$$
\left\{\begin{aligned}
z_{1}(k)= & z_{1}(k-1)+T_{s} z_{2}(k-1)+T_{s} v(k-1) \\
& +L_{1}^{d}\left[I_{o}(k-1)-z_{1}(k-1)\right], \\
z_{2}(k)= & z_{2}(k-1)+L_{2}^{d}\left[I_{o}(k-1)-z_{1}(k-1)\right],
\end{aligned}\right.
$$

$L^{d}=\left[\begin{array}{ll}L_{1}^{d} & L_{2}^{d}\end{array}\right]^{T}$ is the observer gain of discrete ESO in (8) and its value is given as follows:

$$
L^{d}=\left[\frac{2(1-\beta)(1-\beta)^{2}}{T_{s}}\right]^{T}
$$

where $\beta=e^{-\sigma T_{s}}$. In this paper, we take $\sigma=100$ when designing the extended state observer.

3.2. Online Parameter Estimation. Most of the parameters of the continuous system model have clear physical meaning and the parameters identification methods of the continuous system can be divided into the direct method and indirect method: the direct method is to directly establish the discrete-time model with the same parameters as the continuous-time model and the indirect method is to first discretize the continuous model and then identify the parameters of the discrete model. In this paper, by using the direct method, the following system processes can be obtained according to equation (1):

$$
y=\phi^{T} \theta \text {. }
$$

In equation (10), $\theta$ is parameters vector to be estimated:

$$
\begin{aligned}
& y(k)=\left[I_{o}(k)\right], \\
& \phi(k)=\left[\begin{array}{lll}
-\dot{I}_{o}(k) & u(k)
\end{array}\right]^{T}, \\
& \theta=\left[\begin{array}{ll}
\theta_{1} & \theta_{2}
\end{array}\right]^{T}=\left[\frac{L}{R} \frac{V_{s}}{R}\right]^{T} .
\end{aligned}
$$

A supervisory recursive least square (SRLS) method is proposed to estimate parameters and its schematic block diagram is presented in Figure 5.

In Figure 5, the excitation variance is used to enable RLS estimation, and the estimation error variance is used to trigger parameter updating. Several linear low pass (LP) 


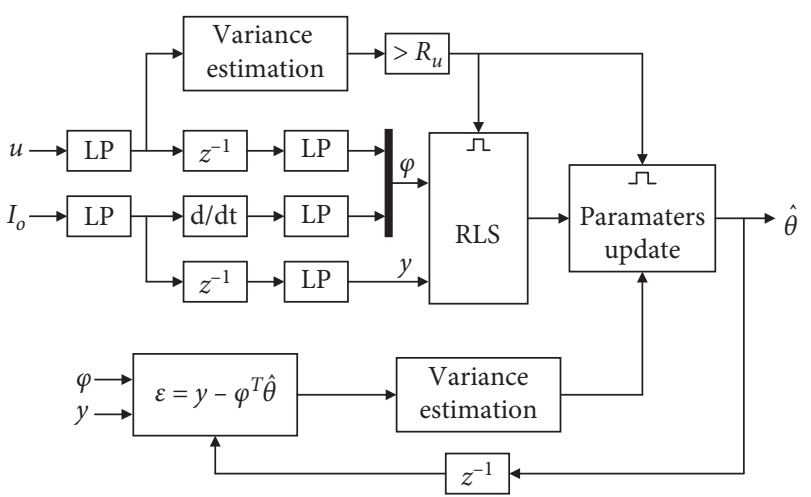

FIgURE 5: The block diagram of SRLS.

filters are adopted to reduce noise interference. The central difference method is used to calculate the differential of $I_{e}$ and its form is presented in equation (14). It can be illustrated that the central difference method has a sampling period delay:

$$
\dot{I}_{o}(k)=\frac{I_{o}(k+1)-I_{o}(k-1)}{2 T_{s}} .
$$

3.2.1. Recursive Least Square Method. The recursive formulas of the recursive least squares method are given in the following equations:

$$
\begin{aligned}
\mathcal{E}(k) & =y(k)-\phi^{T}(k) \widehat{\theta}(k-1), \\
K(k) & =\frac{P(k-1) \phi(k)}{\lambda+\phi^{T}(k) P(k-1) \phi(k)} \\
\widehat{\theta}(k) & =\widehat{\theta}(k-1)+K(k) \varepsilon(k), \\
P(k) & =\frac{1}{\lambda}\left[P(k-1)-K(k)^{T} \phi(k) P(k-1)\right],
\end{aligned}
$$

where $\varepsilon(k)$ is the $k$-step estimation error; $K(k)$ is the recursive least squares gain; $\widehat{\theta}(k)$ is the $k$-step parameters estimation; $P(k)$ is the $k$-step recursive variance; and $\lambda$ is the forgetting factor (0.99).

3.2.2. Triggering Estimator. The variance estimation method of control excitation $u$ proposed in this paper is given in the following equation:

$$
\left\{\begin{array}{l}
\bar{u}(k)=\frac{1}{N} \sum_{i=k-N-1}^{k} u(i), \\
r_{u}(k)=\eta_{u} r_{u}(k-1)+\left(1-\eta_{u}\right)[u(k)-\bar{u}(k-1)][u(k)-\bar{u}(k)],
\end{array}\right.
$$

where $\bar{u}(k)$ represents the mean of the excitation $u$ and $N$ is the window size; $r_{u}(k)$ is the variance estimate of the excitation $u$; and $\eta_{u} \in(0,1)$ is the adjustment coefficient and the smaller the value of $\eta_{u}$, the faster the update will be. In this paper, $\eta_{u}$ is 0.95 .

The result of variance estimation of step excitation is presented in Figure 6. From Figure 6, it can be seen that when the signal steps, the variance of estimation will change abruptly, and when the signal is stable, the variance of estimation will tend to 0 . The variance estimation result of random excitation is given in Figure 7. It is shown in Figure 7 that when the variance of signal increases, the variance estimation result also increases. Compared with the methods in $[12,14]$, the variance estimating method of the excitation signal given in equation (19) is suitable for random excitation, when it is used as the excitation detection to enable the parameter estimator.

When the excitation variance $r_{u}$ is greater than the set threshold $R_{u}$, it indicates that the excitation is sufficient, and then the RLS estimator is executed; when the variance estimate is less than the set threshold, it means that the excitation is too small to fully excite the system and the parameter estimator will be stopped.

3.2.3. Parameter Updating. In this paper, the variance of estimation error is used as the condition of parameter updating. The variance estimation $[23,25]$ of the estimation error $\mathcal{E}$ is given in

$$
r_{\varepsilon}(k)=\eta_{\varepsilon} r_{\varepsilon}(k-1)+\left(1-\eta_{\varepsilon}\right) \varepsilon^{2}(k),
$$

where $r_{\varepsilon}(k)$ is the variance estimate of $\varepsilon(k)$ and $\eta_{\varepsilon} \in(0,1)$ is the adjustment coefficient which is 0.95 here.

In the parameter estimation period, when the variance estimate of $\varepsilon(k)$ is less than the set threshold $R_{\varepsilon}$, the parameter estimate will be updated; otherwise, the parameter estimates remain unchanged. The simulation results of parameter estimation are shown in Figure 8. "PE flag" in Figure 8 means "parameters estimating flag." The value of 1 means the estimator works; otherwise, the estimator does not work. It is indicated in Figure 8 the results of parameter estimation are in good agreement with the actual values.

Also, in order to ensure the positive definiteness of the variance matrix $P(k)$, we can use the square root algorithm or UD decomposition algorithm [26], which will not be discussed in this paper.

3.2.4. Failure Analysis. Online parameter identification can be used not only to adjust controller parameters but also to judge system faults. Table 2 gives the failure analysis.

Since the PWM method is used to adjust the current, when the coil inductance is very small (circuit short), the current control will not work. The circuit current will oscillate seriously and even damage the circuit itself and other devices. Therefore, the above online parameters are of great significance for fault diagnosis.

\subsection{Differential Feedforward with Tracking Differentiator.} The form of tracking differentiator used in this paper is given as follows: 


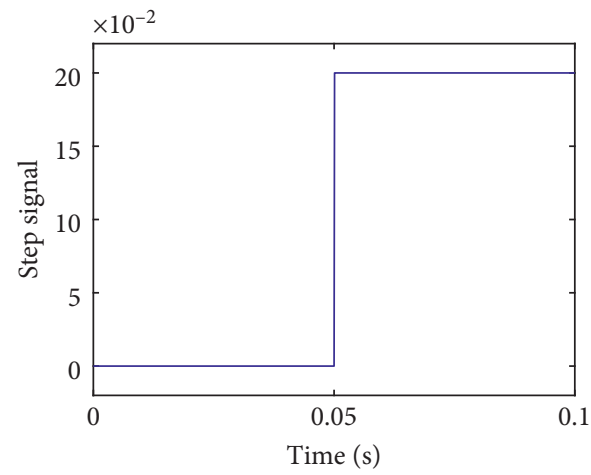

(a)

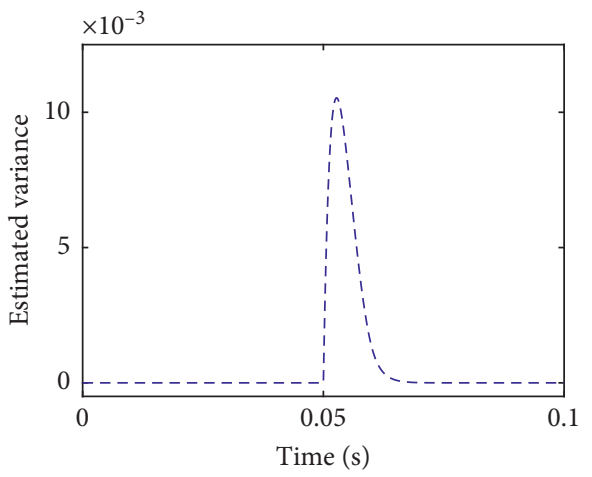

(b)

FIGURE 6: Variance estimation output of the step signal.

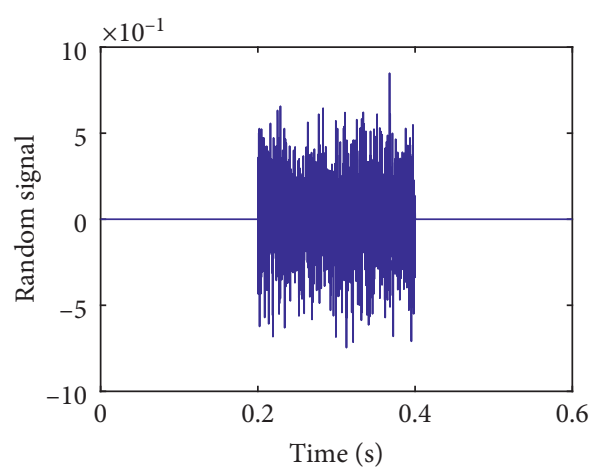

(a)

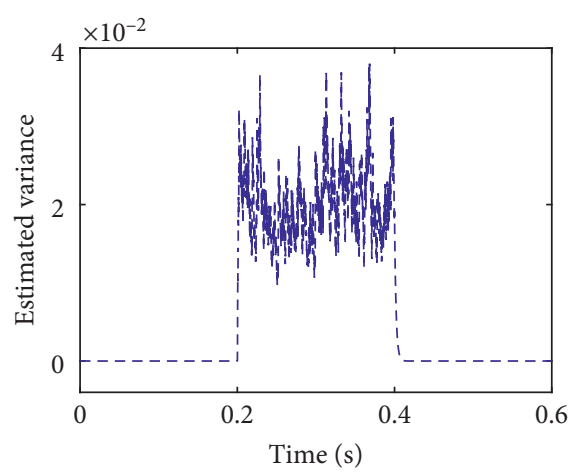

(b)

FIgURE 7: Variance estimation output of the random signal.

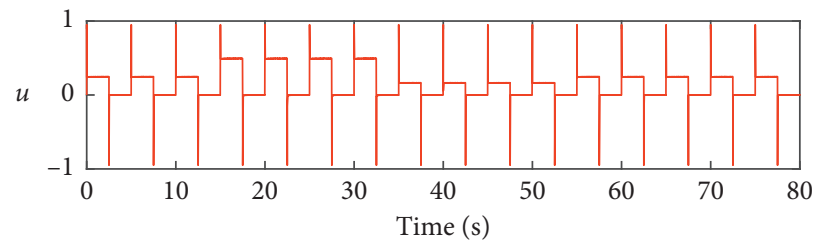

(a)

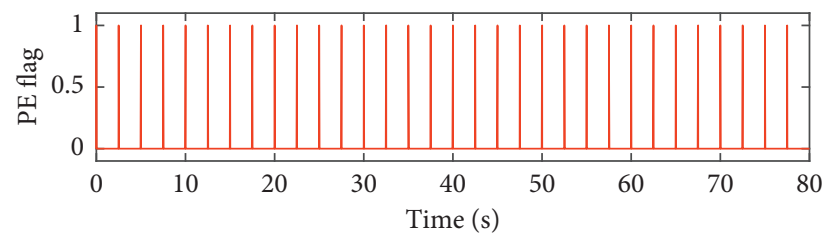

(b)

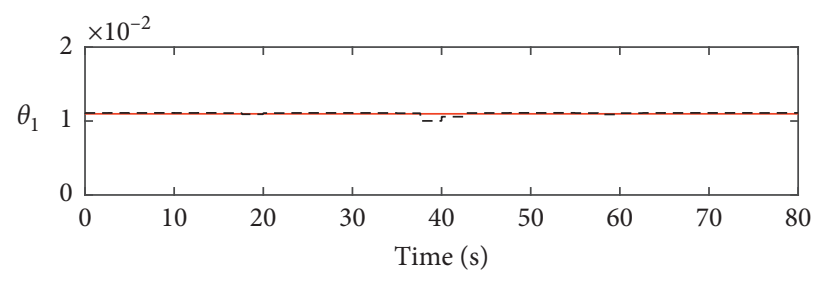

$-\theta_{1}$

$--\hat{\theta}_{1}$ SRLS

(c)

Figure 8: Continued. 


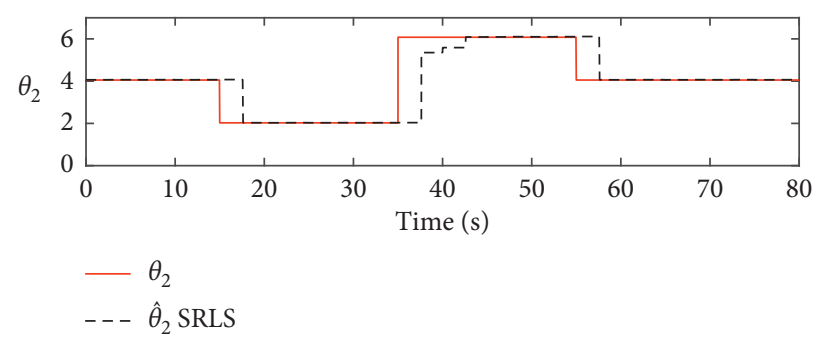

(d)

FIGURE 8: Parameters estimation results.

TABle 2: Failure analysis.

\begin{tabular}{lccc}
\hline$\theta_{2} \theta_{1}$ & Too small & Normal & Too large \\
\hline Too small & Circuit open & Partial short & Circuit short \\
Normal & Power undervoltage & $\checkmark$ & Circuit short \\
Too large & & & Circuit short \\
\hline
\end{tabular}

$$
\left\{\begin{array}{l}
x_{1}(k)=x_{1}(k-1)+T_{s} x_{2}(k-1) \\
x_{2}(k)=x_{2}(k-1)+T_{s} f_{h} \\
f_{h}=\text { flan }\left(x_{1}-x_{r}, x_{2}, r, h_{0}\right)
\end{array}\right.
$$

where $x_{r}$ is the signal input of TD; $x_{1}$ and $x_{2}$ are the state variables of TD, $x_{1}$ is the tracking signal of $x_{r}$ and $x_{2}$ is the differential signal; and $T_{s}$ represents the system sampling period $(0.1 \mathrm{~ms})$. flan $(\cdot)$ is the fastest control synthesis function which can improve the convergence speed of the system and eliminates the high frequency oscillation when the signal is stable. The fastest control integrated function is denoted by $f_{h}=\operatorname{flan}\left(\xi_{1}, \xi_{2}, r, h\right)$, and its function form is given by equation (22).

In equation (22), $r$ is the fast factor, and the larger the $r$ value, the stronger the signal tracking ability. $h$ is the filtering factor, and the larger the $h$ value, the stronger the suppression of noise. In this paper, $r=10^{6}$ and $h=10 T_{s}$ are adopted:

$$
\left\{\begin{array}{l}
d=r h, \\
d_{0}=h d, \\
y=\xi_{1}+h \xi_{2}, \\
a_{0}=\sqrt{d^{2}+8 r|y|}, \\
a= \begin{cases}\xi_{2}+\frac{a_{0}-d}{2} \operatorname{sign}(y), & |y|>d_{0}, \\
\xi_{2}+\frac{y}{h}, & |y| \leq d_{0},\end{cases} \\
f_{h}=- \begin{cases}r \cdot \operatorname{sign}(a), & |y|>d, \\
r \cdot \frac{a}{d}, & |y| \leq d .\end{cases}
\end{array}\right.
$$

When the input signal is a unit step signal, the tracking differentiator output is shown in Figure 9. And, when the input signal is a sinusoidal signal $(10 \mathrm{~Hz})$, the output is shown in Figure 10. The results illustrate that the tracking differentiator has a good tracking and differential performance for the input signal.

When the system only uses differential feedforward control, the transfer equation of the system is given as follows:

$$
T_{I_{o}-u}^{F F}(s)=\frac{I_{o}(s)}{I_{e}(s)}=\frac{K_{f}\left(V_{s} / R\right) s}{1+(L / R) s} .
$$

According to the amplitude frequency characteristics of the transfer function in equation (23), if equation (24) is true, the system gain $\left|T_{I_{o}-u}^{F F}(s)\right|=1$ at high frequency input:

$$
K_{f} \frac{V_{s}}{R}=\frac{L}{R}
$$

Then,

$$
K_{f}=\frac{\theta_{1}}{\theta_{2}} .
$$

Due to the limitation of the frequency band of the differentiator, the value of $K_{f}$ is slightly less than the above value to reduce the overshoot.

3.4. Proportional Control Gain Adjustment. If the system only adopts an error proportional controller, namely, $u=K_{p} e$, the transfer function of system error is given in

$$
\frac{E(s)}{I_{e}(s)}=\frac{1+(L / R) s}{1+\left(\left(K_{p} V_{s}\right) / R\right)+(L / R) s} .
$$

The larger the value of $K_{p}$, the faster the response speed of the closed-loop system and the smaller the static error for tracking step signal. But, in practical application, too large $K_{p}$ value will make the closed-loop system oscillate.

According to equation (26), a closed-loop eigenvalue can be easily obtained:

$$
\sigma=-\frac{1+\left(\left(K_{p} V_{s}\right) / R\right)}{(L / R)}
$$

A simple gain adjustment method is adopted in

$$
\sigma=\text { const. }
$$




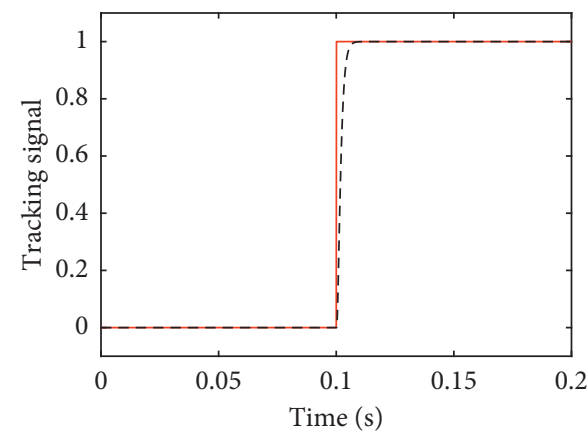

$\begin{array}{ll}- & x_{r} \\ --- & x_{1}\end{array}$

(a)

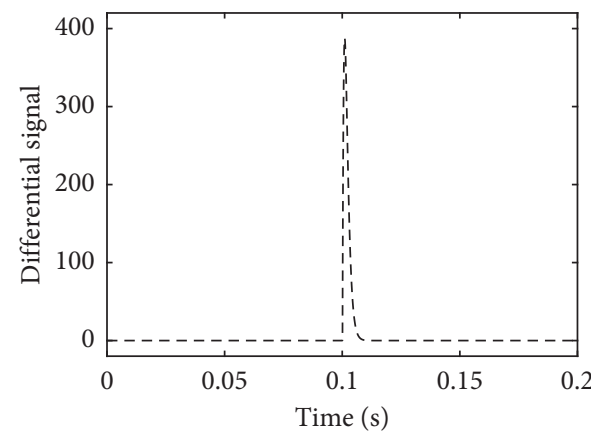

(b)

FIgURE 9: TD output results for step input.

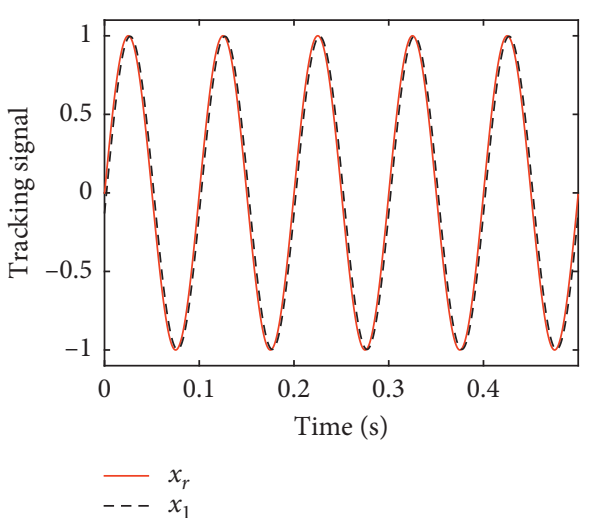

(a)

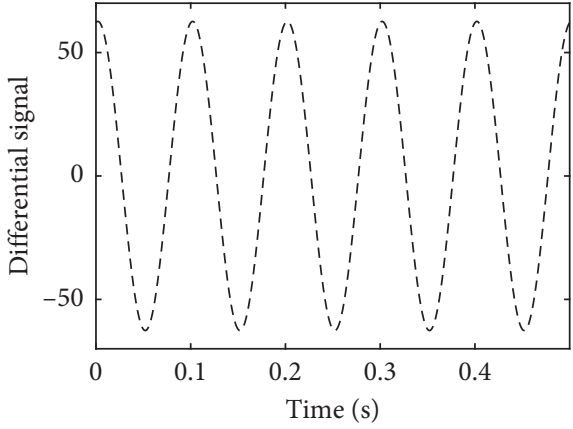

(b)

FIgURE 10: TD output results for sine input $(10 \mathrm{~Hz})$.

In this paper, we take $\sigma=-642$ according to experiment results; then,

$$
K_{p}=-\frac{1+\sigma \theta_{1}}{\theta_{2}}
$$

3.5. Stability Analysis. The system controller consists of three parts: differential feedforward, proportional error control, and ESO compensation. Feedforward control is introduced to improve the response speed of the system without affecting the stability of the system.

Due to the separation design characteristics of ADRC, the stability of proportion control and ESO can be discussed separately. For the error proportional control, when the real part of the characteristic root of the closed-loop system is less than 0 , for the ESO, according to the extremely low configuration method in the literature, the stability of the linear ESO can be guaranteed. Therefore, it is not difficult to get the stability of the controller designed in this paper.

Meanwhile, due to the separable design characteristics of each module of ADRC, the stability of proportional control and ESO can be discussed separately when discussing system stability. For the error proportional control, when the real part of the closed-loop system in equation (26) is less than 0 , the system is stable. For ESO, the linear ESO is guaranteed to be stable according to the pole placement method in [24]. Therefore, it is not difficult to find the stability of the controller designed in this paper.

\section{Experiment Results and Comparisons}

4.1. The Structure of Experiment System. The current driver is mainly composed of a power supply circuit, microcontroller unit (MCU), bootstrap circuit, and $\mathrm{H}$-bridge drive circuit. The circuit board designed in this paper is shown in Figure 11.

MCU uses STM32F401 of ST Company and bootstrap circuit chip selects IR2110 of IR Company. Pulse width modulation (PWM) control is used in $\mathrm{H}$-bridge drive circuit. The main frequency of $\mathrm{MCU}$ is $84 \mathrm{MHz}$, which generates $84 \mathrm{KHz}$ PWM wave with a resolution of $1 / 1000$.

The experimental system consists of a rapid control prototype, a drive circuit board, and a magnetorheological damper as shown in Figure 12.

In Figure 12, Speedgoat (type: IO135, Speedgoat ${ }^{\circledR}$, Switzerland), as a rapid control prototype, is used to execute control programs and record signal data. 

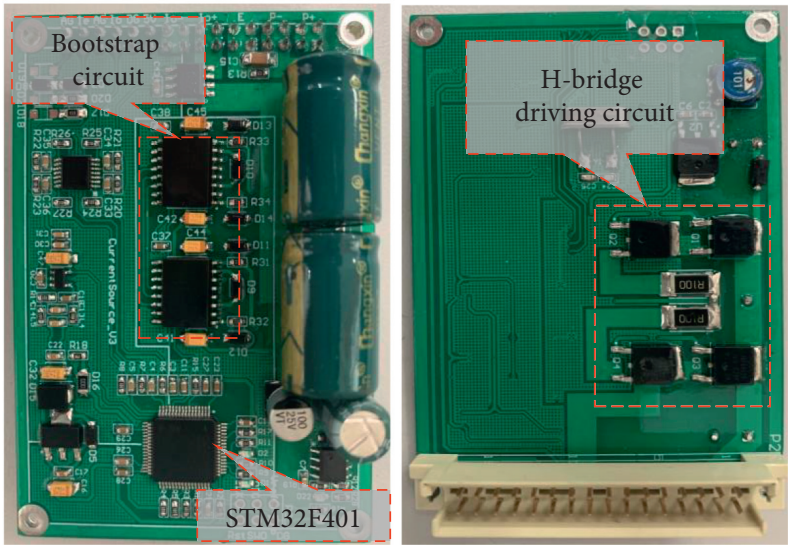

Figure 11: PCB circuit board.

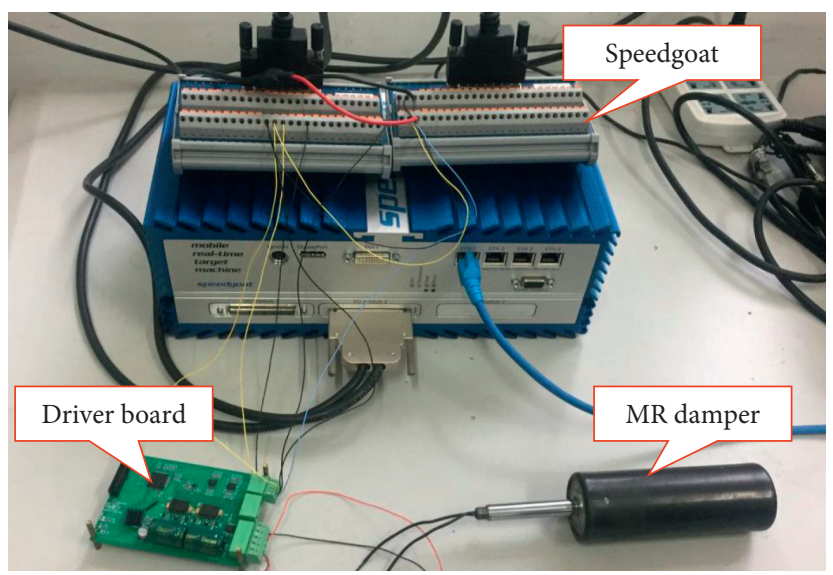

Figure 12: The experiment system setup.

4.2. The Experiment Results of Online Parameter Estimation. In order to test the effect of an online parameter estimator, two experiments are designed in this paper: one is changing the supply voltage and the other is changing the loop load. The figure shows the online parameter estimation results of the first experiment. When the driver works, the power supply voltage changes from $24 \mathrm{~V}$ to $36 \mathrm{~V}$. It is not difficult to see from equation (12) that the parameter value $\theta_{2}$ will increase significantly with the power supply voltage $V_{s}$. The results of parameter estimation are given in Figure 13 and the estimated parameters are in good agreement with the theoretical values.

Figure 14 shows the experimental diagram of load changing. At the beginning of the experiment, two identical loads are connected in series in the circuit, and one of them is shorted in the experiment so that the total load of the circuit changes. It is not difficult to see from equation (12) that the parameters will increase obviously, and the parameter estimation results are shown in Figure 15. Figure 15(a) shows that excitation is the square-wave signal and variance estimation of excitation is given in Figure 15(b). From Figures 15(c) and 15(d), the estimated results and the theoretical values are in good agreement.
4.3. Comparisons between PI and ADRC. The results comparisons between PI and ADRC are shown in Figures 16 and 17. Figure 16 shows the results of the system under step input (1 A). From Figure 16, because of applying ESO, the output of the system can also track step signals quickly without static error when only proportional feedback control is used. However, the ESO does not have any contribution to system response speed compared with the PI controller. It is also obvious in Figure 17 that both systems using PI and ADRC have phase lag when tracking the sinusoidal signal.

4.4. Comparisons between $A D R C$ and $D F-A D R C$. The results comparisons between ADRC and DF-ADRC are shown in Figures 18 and 19. The differential feedforward control with TD is introduced into the controller design to further improve the response speed of the system and it works both in step input and sinusoidal input (Table 3). When comparing ADRC with DF-ADRC, it is not hard to see that the differential feedforward (DF) is the main driving force of system response speed improvement. 


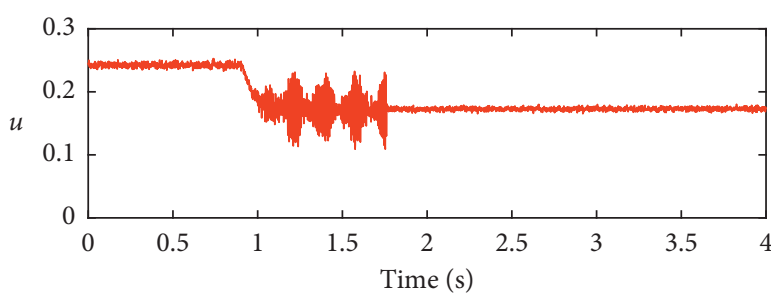

(a)

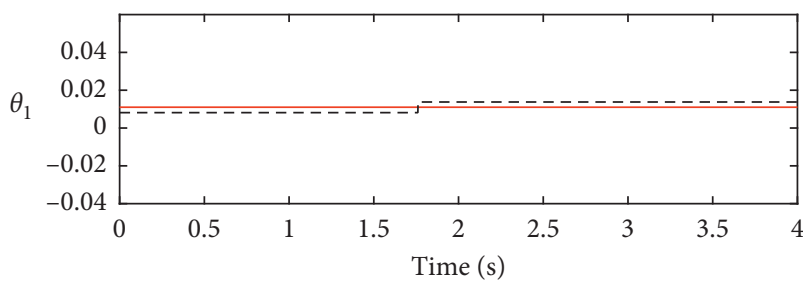

$-\theta_{1}$

$---\hat{\theta}_{1}$ SRLS

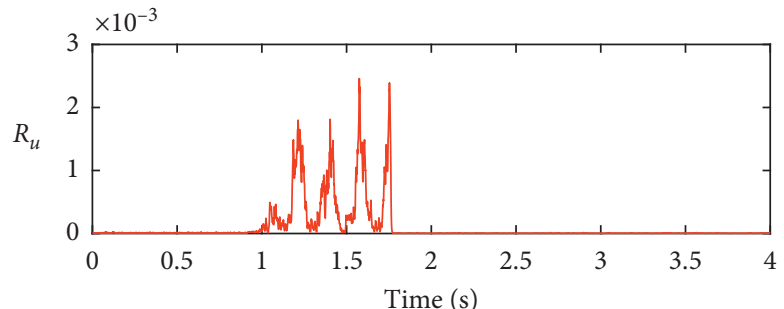

(b)

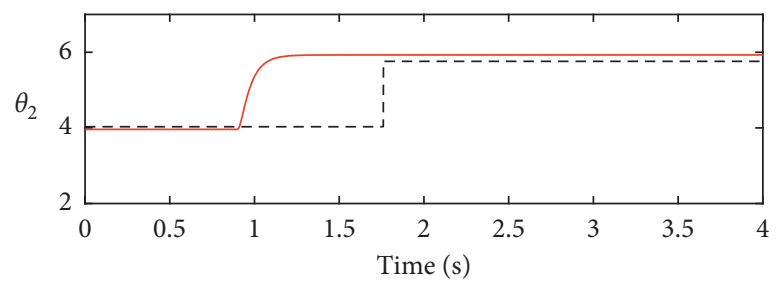

$-\theta_{2}$

$---\hat{\theta}_{2}$ SRLS

(c)

(d)

FIgURE 13: Parameter estimation results with source voltage changing.

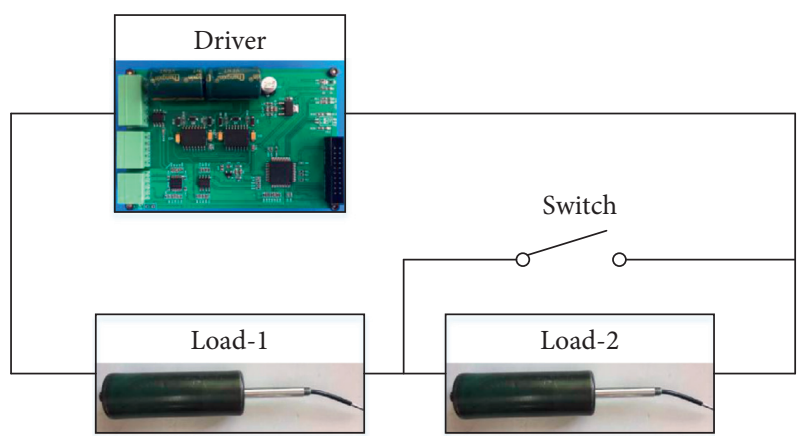

FIGURE 14: Load changing experimental diagram.

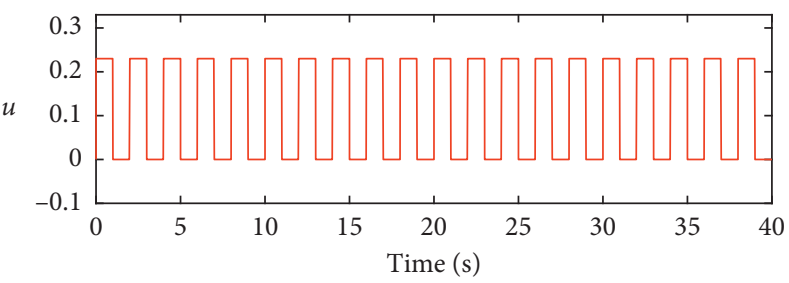

(a)

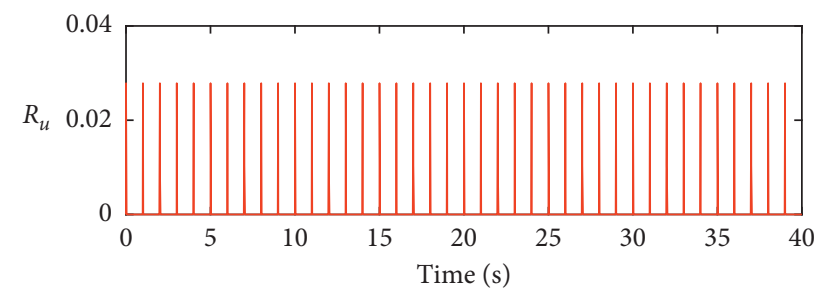

(b)

FIgURE 15: Continued. 


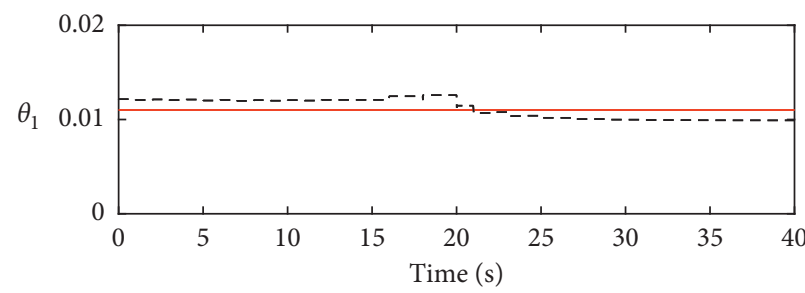

$-\theta_{1}$

$---\hat{\theta}_{1}$ SRLS

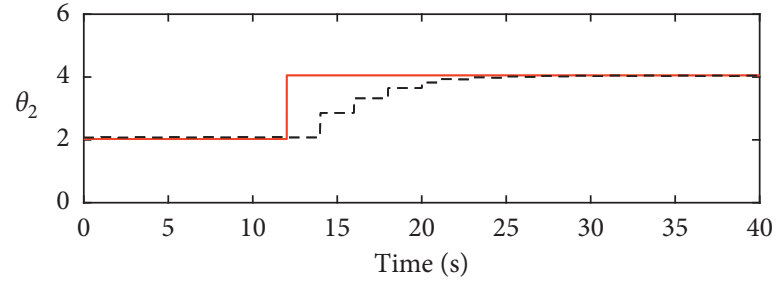

$-\theta_{2}$

$--\hat{\theta}_{2}$ SRLS

(c)

(d)

FIGURE 15: Parameter estimation results with load changing.

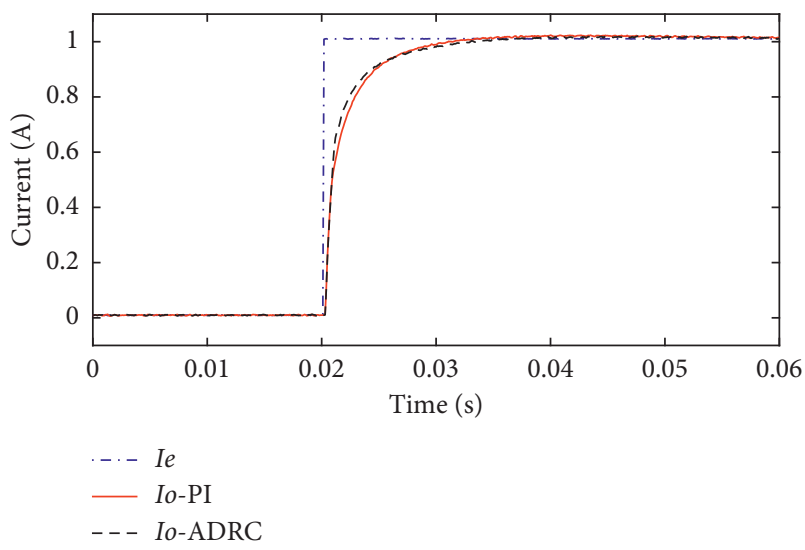

FIgURE 16: The results comparisons between PI and ADRC under step input (1 A).

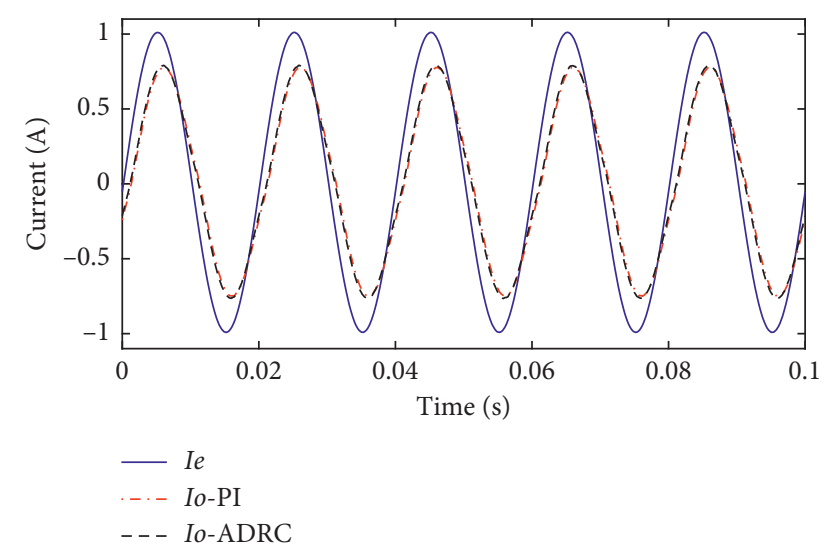

FIgURE 17: The results comparisons between PI and ADRC under sinusoidal input $(1 \mathrm{~A}, 50 \mathrm{~Hz})$.

4.5. Comparisons between PI and AADRC. Figure 20 shows the experimental results of the PI controller when the power supply voltage changes and the results of the AADRC controller are presented in Figure 21. By comparison, it is not difficult to find that when the power supply voltage increases from $24 \mathrm{~V}$ to $36 \mathrm{~V}$, the output of the system using PI controller oscillates continuously and the results are unsatisfactory; the output of the system using AADRC

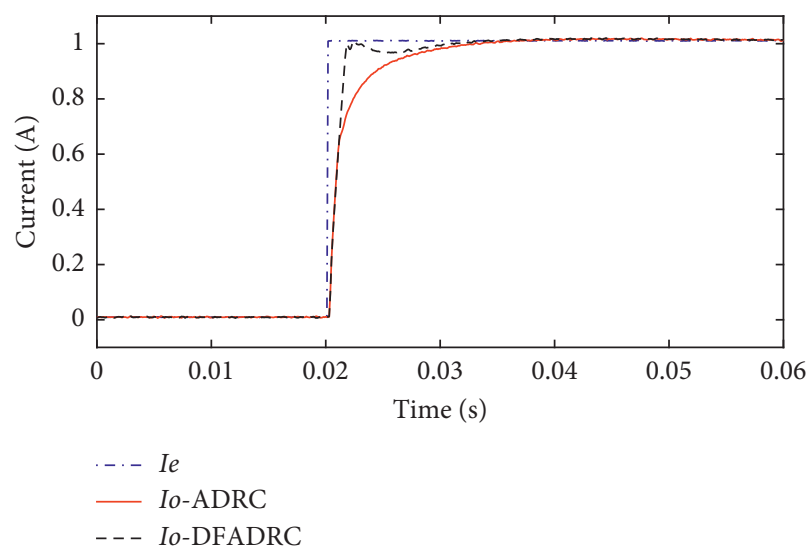

FIgURE 18: The results comparisons between ADRC and TDADRC under step input $1 \mathrm{~A}$.

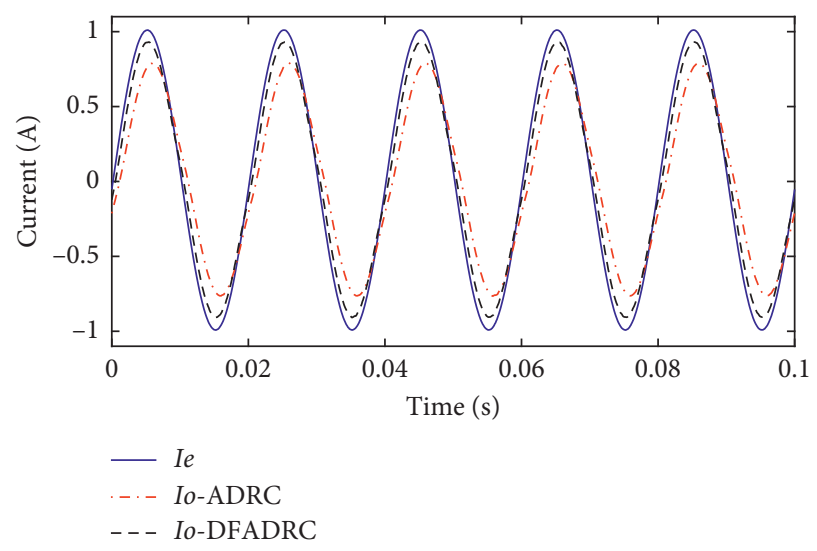

FIgURE 19: The comparison of results between ADRC and TDADRC under sinusoidal input $(1 \mathrm{~A}, 50 \mathrm{~Hz})$.

TABLE 3: Step input response of ADRC and DF-ADRC.

\begin{tabular}{lcc}
\hline Method & Rise time $(\mathrm{ms})$ & Fall time $(\mathrm{ms})$ \\
\hline ADRC & 4 & 4.4 \\
DF-ADRC & 1.2 & 1 \\
\hline
\end{tabular}

controller restores steady after a short period of oscillation. This is because, with the increase of the power supply 

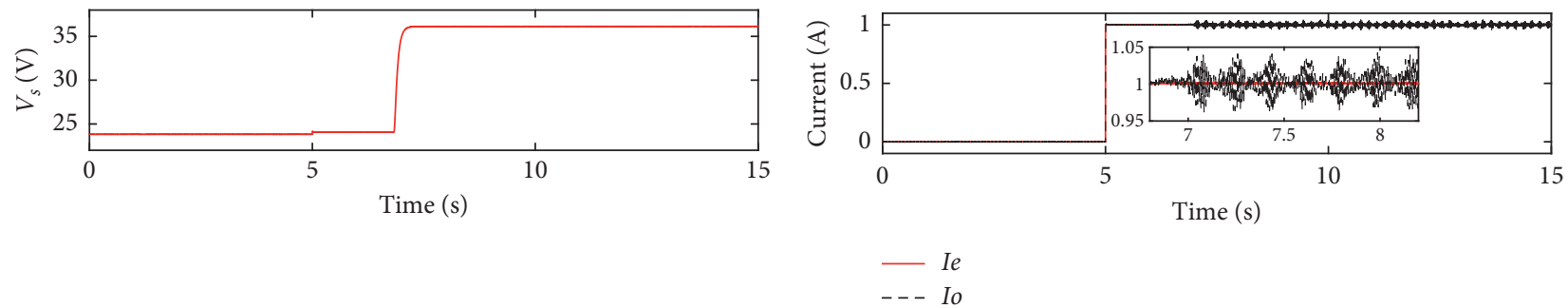

(a)

(b)

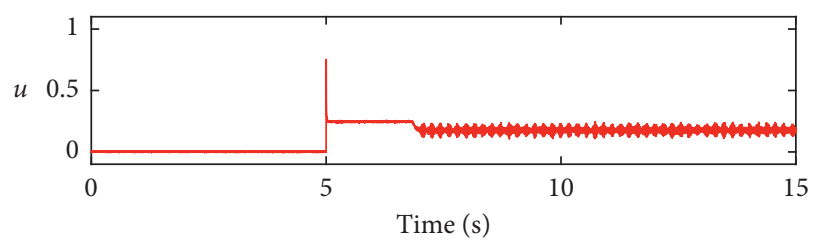

(c)

FIgURE 20: The results of the system using PI controller with $V_{s}$ changing.
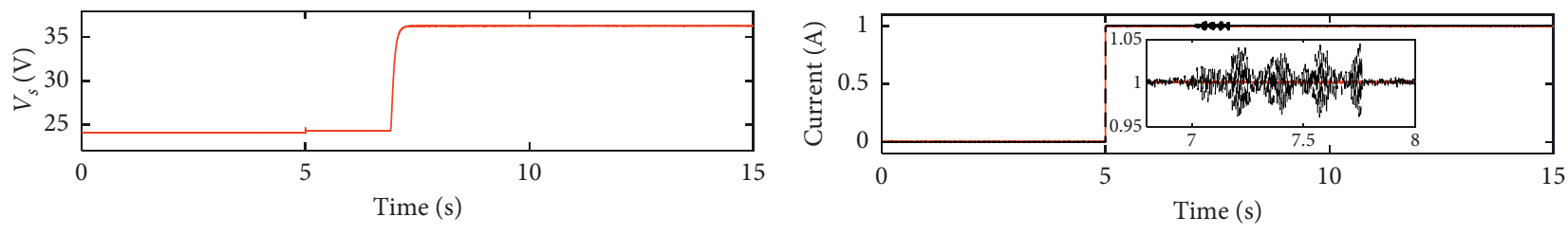

$$
-I e
$$

(a)

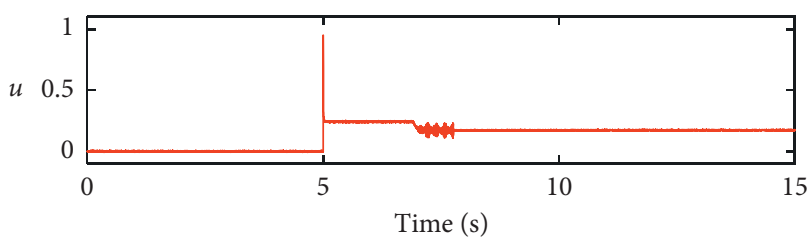

(b)

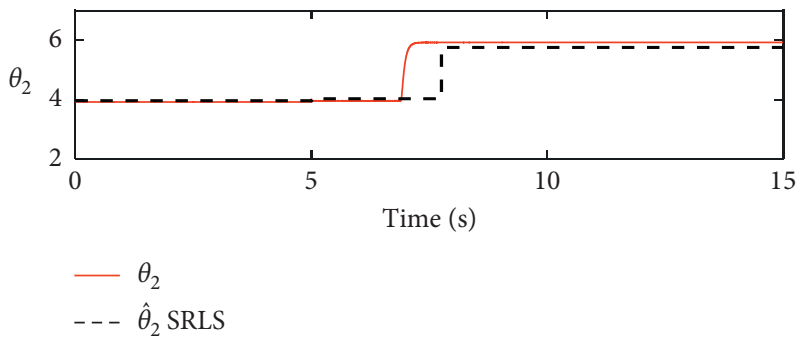

(c)

(d)

FIgUre 21: The results of the system using AADRC with $V_{s}$ changing.

voltage, the system parameters change and the system output oscillates. At this time, the variance of the system control excitation $u$ becomes larger and the online parameter estimator works when the threshold condition of excitation variance is satisfied. Since proportional control gain $K_{p}$ is adjusted by the parameters updated, the system output tends to be steady again.

\section{Conclusion}

An adaptive active disturbance rejection controller is used in the current driver design of the MR damper. Because of applying ESO, the output of the system can also track step signals quickly without static error when only the proportional feedback control is used. Also, the efficiency of setting control parameters is greatly improved by using ADRC separation design characteristics. A supervisory recursive least square (SRLS) method is proposed for real-time parameter estimation. The variance estimation method of control excitation is proposed to trigger the estimator. That is, to guarantee that the estimator is performed only there is enough excitation. Thus, the parameter adaptability of the closed-loop system is improved by adopting AADRC proposed in this paper.

\section{Data Availability}

The experimental data used to support the finding of this study are all included in the manuscript.

\section{Conflicts of Interest}

The authors declare that they have no conflicts of interest. 


\section{Acknowledgments}

The research was supported by the Science Challenge Project (no. Tz2016006-0104) and Natural Science Foundation of China Government (no. 11472135).

\section{References}

[1] F. A. Shirazi, J. Mohammadpour, K. M. Grigoriadis, and G. Song, "Identification and control of an MR damper with stiction effect and its application in structural vibration mitigation," IEEE Transactions on Control Systems Technology, vol. 20, no. 5, pp. 1285-1301, 2012.

[2] W. Rafajowicz, J. Wickowski, P. Moczko, and E. Rafajowicz, "Iterative learning from suppressing vibrations in construction machinery using magnetorheological dampers," Automation in Construction, vol. 119, Article ID 103326, 2020.

[3] M.-G. Yang, Z.-Q. Chen, and X.-G. Hua, "An experimental study on using MR damper to mitigate longitudinal seismic response of a suspension bridge," Soil Dynamics and Earthquake Engineering, vol. 31, no. 8, pp. 1171-1181, 2011.

[4] G. Z. Yao, F. F. Yap, G. Chen, W. H. Li, and S. H. Yeo, "MR damper and its application for semi-active control of vehicle suspension system," Mechatronics, vol. 12, no. 7, pp. 963-973, 2002.

[5] C. Poussot-Vassal, C. Spelta, O. Sename, S. M. Savaresi, and L. Dugard, "Survey and performance evaluation on some automotive semi-active suspension control methods: a comparative study on a single-corner model," Annual Reviews in Control, vol. 36, no. 1, pp. 148-160, 2012.

[6] M. A. Demetriou, K. Ito, and R. C. Smith, "Adaptive techniques for the MRAC, adaptive parameter identification, and on-line fault monitoring and accommodation for a class of positive real infinite dimensional systems," International Journal of Adaptive Control \& Signal Processing, vol. 23, pp. 193-215, 2010.

[7] Q. Zhang, J. Luo, and L. Wan, "Parameter identification and synchronization of uncertain general complex networks via adaptive-impulsive control," Nonlinear Dynamics, vol. 71, no. 1-2, pp. 353-359, 2013.

[8] J. E. Parkum, N. K. Poulsen, and J. Holst, "Recursive forgetting algorithms," International Journal of Control, vol. 55, no. 1, pp. 109-128, 1992.

[9] B. Wittenmark, "Practical issues in the implementation of self-tuning control," Automatica, vol. 20, no. 5, pp. 595-605, 1984.

[10] I. Åström, L. Ljung, and T. Söderström, "Identification of processes in closed loop-identifiability and accuracy aspects," Automatica, vol. 13, no. 1, pp. 59-75, 1977.

[11] H. Kurz and R. Isermann, "Methods for on-line process identification in closed loop," IFAC Proceedings Volumes Identification in Closed Loop, vol. 8, no. 1, pp. 242-255, 1975.

[12] T. Hägglund and K. J. Åström, "Supervision of adaptive control algorithms," Automatica, vol. 36, no. 8, pp. 1171-1180, 2000.

[13] R. Isermann and K.-H. Lachmann, "Parameter-adaptive control with configuration aids and supervision functions," Automatica, vol. 21, no. 6, pp. 625-638, 1985.

[14] P. E. Moraal, "Adaptive compensation of fuel dynamics in an SI engine using a switching EGO sensor," in Proceedings of IEEE Conference on Decision \& Control, IEEE, Las Vegas, NV, USA, December 2002.

[15] H. Hjalmarsson, "From experiment design to closed-loop control," Automatica, vol. 41, no. 3, pp. 393-438, 2005.
[16] B. Sun and Z. Gao, "A DSP-based active disturbance rejection control design for a $1-\mathrm{kW} \mathrm{H}$-bridge DC-DC power converter," IEEE Transactions on Industrial Electronics, vol. 52, no. 5, pp. 1271-1277, 2005.

[17] K. Sun, Z.-L. Xu, K. Gai, J.-Y. Zou, and R.-Z. Dou, "A novel position controller of PMSM servo system based on activedisturbance rejection controller," in Proceedings of the Proceedings of the Chinese Society of Electrical Engineering, pp. 43-46, Zhongguo Dianji Gongcheng Xuebao, China, November 2007.

[18] Q. Zheng, L. Dong, D. H. Lee, and Z. Gao, "Active disturbance rejection control for MEMS gyroscopes," in Proceedings of the American control conference, pp. 4425-4430, IEEE, Seattle, WA, USA, June 2008.

[19] H. Sira-Ramírez, C. López-Uribe, and M. Velasco-Villa, "Linear observer-based active disturbance rejection control of the omnidirectional mobile robot," Asian Journal of Control, vol. 15, pp. 51-63, 2013.

[20] J. Yao and W. Deng, "Active disturbance rejection adaptive control of hydraulic servo systems," IEEE Transactions on Industrial Electronics, vol. 64, no. 10, pp. 8023-8032, 2017.

[21] J. Han, Active Disturbance Rejection Control Technique-The Technique for Estimating and Compensating the Uncertainties, National Defense Industry Press, Beijing, China, 2008.

[22] J. Han, "From PID to active disturbance rejection control," IEEE Transactions on Industrial Electronics, vol. 56, no. 3, pp. 900-906, 2009.

[23] R. Isermann and M. Münchhof, Identification of Dynamic Systems: An Introduction with Applications, Springer Science \& Business Media, Berlin, Germany, 2010.

[24] R. Miklosovic, A. Radke, and Z. Gao, "Discrete implementation and generalization of the extended state observer," in Proceedings of the American control conference, IEEE, Seattle, WA, USA, June 2006.

[25] T. Hägglund, New Estimation Techniques for Adaptive Control, Lund University, Lund, Sweden, 1983.

[26] G. J. Bierman, Factorization Methods for Discrete Sequential Estimation, Courier Corporation, Chelmsford, MA, USA, 2006. 